\title{
Revue des connaissances sur la micro-filière ovine laitière en Tunisie : état des lieux et perspectives de relance de la race sicilo-sarde
}

\author{
Rafik Aloulou ${ }^{(1)}$, Pierre-Guy Marnet ${ }^{(2)}$, Youssef M'Sadak ${ }^{(1)}$ \\ (1) Université de Sousse. Institut Supérieur Agronomique. BP 47. TN-4042 Chott-Mariem, Sousse (Tunisie). E-mail : \\ aloulou1965@yahoo.fr \\ ${ }^{(2)}$ Agrocampus Ouest. Département Production Animale, Agro-Alimentaire et Nutrition. FR-35042 Rennes (France).
}

Reçu le 13 février 2018, accepté le 12 juin 2018, mis en ligne le 21 juin 2018.

Cet article est distribué suivant les termes et les conditions de la licence CC-BY (http://creativecommons.org/licenses/by/4.0/ deed.fr)

Introduction. La race sicilo-sarde est la seule race ovine laitière tunisienne. Elle est à la base d'une micro-filière de production de fromages. Son effectif s'est effondré dans les années 1990 en raison de choix économiques et politiques peu favorables ainsi que d'un manque de maitrise technique des élevages, mettant la pérennité de cette filière en danger. Toutefois, des initiatives pour la préserver sont entreprises depuis quelques années. Cette revue de la littérature, scientifique et professionnelle, fait le point sur les connaissances actuelles de la filière laitière ovine et présente les actions en cours ou à entreprendre en faveur de sa sauvegarde.

Littérature. La race sicilo-sarde affiche des niveaux de production laitière faibles mais améliorables, même dans des conditions extensives. Les conduites d'élevage (allaitement/sevrage/traite) et de reproduction devraient être revues afin d'accroitre la production laitière. De même, la voie génétique devrait être mobilisée par la mise en place d'un schéma de sélection appuyé sur le relevé de phénotypes d'intérêt en ferme (quantité et qualité de lait, morphologie mammaire) et/ou par introduction de sang sarde en recourant à l'insémination artificielle.

Conclusions. Un regain d'intérêt pour la race sicilo-sarde en Tunisie est constaté depuis 2005, à travers une réorganisation de la filière, insufflant un nouveau dynamisme au sein de la profession. Les marges importantes de progrès ont été mises en évidence, tant au niveau des animaux que des éleveurs. L'aval de la filière doit aussi aider à une meilleure identification des produits et du terroir de production auprès des consommateurs. Ainsi se dessineront de nouvelles opportunités de développement de cette micro-filière, seule à même d'assurer sa durabilité.

Mots-clés. Ovin, sicilo-sarde, aptitude laitière, mutations, durabilité, Tunisie.

Review of the Sicilo-Sarda dairy sheep micro-sector in Tunisia: current situation and prospects for the recovery of the Sicilo-Sarda breed

Introduction. The Sicilo-Sarda is the only Tunisian dairy sheep breed. Milk from this breed is used in a micro-sector of cheese production. Numbers of sheep from this breed fell significantly during the 1990s, due to unfavorable economic and political choices, as well as a lack of technical control of livestock, which risked the sustainability of this cheese production sector. However, initiatives to preserve this breed have taken place in recent years. The present review of the scientific and professional literature highlights the current knowledge of the sheep dairy sector in Tunisia and presents the actions currently under way or to be undertaken in favor of its safeguarding.

Literature. The Sicilo-Sarda breed shows low levels of milk production but this can be improved even in extensive systems. Breeding (suckling/weaning/milking) and reproductive management needs to be reviewed in order to increase milk production. Similarly, the breed's genetic pathway needs to be mobilized through the establishment of a selection scheme based on farm phenotypes of interest (quantity and quality of milk, mammary morphology) and/or the introduction of Sardinian blood via artificial insemination.

Conclusions. A renewed interest in the Sicilo-Sarda breed in Tunisia has been noted since 2005 through a reorganization of the sector, giving new dynamism to the profession. Significant margins of progress have been highlighted, both in terms of animals and breeders. The downstream sector of this milk chain must also help create a better identification of the products and the "terroir" of production for consumers. This will create new opportunities to develop this micro-sector to ensure its sustainability.

Keywords. Sheep, Sicilo-Sarda, dairy performance, mutations, sustainability, Tunisia. 


\section{INTRODUCTION}

Les petits ruminants, notamment les ovins, représentent une part importante des ressources animales en Tunisie. Ils sont représentés par plusieurs races ou populations autochtones devenues, à travers plusieurs générations, rustiques et parfaitement adaptées à leur environnement. Le cheptel ovin tunisien compte environ 3,8 millions d'Unités Femelles (OEP, 2016). Il est constitué principalement de trois races à viande : barbarine $(65 \%)$, Queue Fine de l'Ouest $(32 \%)$ et noire de Thibar $(2 \%)$.

Bien qu'il occupe une place marginale dans la filière laitière, l'élevage ovin laitier représente une microfilière utilisant quasi-exclusivement la race sicilo-sarde, seule race ovine laitière spécialisée en Afrique du Nord. Son effectif représente moins de $0,5 \%$ de l'effectif total des femelles et a été en régression très forte et quasi en extinction ces dernières années. La race sicilo-sarde est d'ailleurs considérée par la Banque Nationale de Gènes de Tunisie (BNG, 2018) comme prioritaire en matière de conservation des ressources génétiques animales autochtones menacées. Elle est considérée comme ayant un intérêt socio-économique considérable, notamment en tant qu'outil de développement durable des zones rurales. Les produits typiques et artisanaux à base de lait de brebis sicilo-sarde (ricotta et sicilien) sont à valeur ajoutée certaine mais ne sont pas connus de tous les consommateurs. La consommation de ces produits laitiers ovins, non encore protégés, n'a pour le moment qu'une portée régionale. Ils ont cependant un rôle important à travers les composantes du paysage et de la culture populaire qu'ils véhiculent.

Cette race pâture les vallées et les collines des régions montagneuses et subhumides entre Béja et Bizerte (Nord de la Tunisie) qui sont déjà des zones difficiles. Elle présente en plus un niveau de production faible conditionné par un mode de conduite traditionnel et souvent extensif, ce qui ne favorise pas cette race dans le choix des éleveurs qui en dégagent de trop faibles revenus. En effet, la production laitière moyenne de cette race reste toujours plus faible que celle des races pures dont elle dérive, la sarde et la comisana (Haenlein, 2007 ; Piras et al., 2007). Cependant, cette race affiche des traits uniques comme la résistance aux maladies ou la tolérance aux conditions climatiques extrêmes. Les générations futures pourraient en avoir besoin pour affronter les enjeux des changements climatiques, de l'émergence de nouvelles maladies et de la demande croissante de produits de l'élevage (FAO, 2007). Outre la voie de la conservation pure, une bonne caractérisation et une valorisation plus rationnelle des produits restent les principales alternatives pour améliorer la productivité de la race et remédier ainsi à la régression des effectifs de la race sicilo-sarde en Tunisie.
Cette revue bibliographique vise à faire un état des lieux des connaissances sur cette race sicilo-sarde, à travers une présentation de l'évolution des effectifs, les conduites d'élevage recensées, les performances obtenues ainsi que les principales contraintes auxquelles cette micro-filière est confrontée. Ce tour d'horizon se terminera par une réflexion sur les actions déjà lancées ou qu'il faudrait mettre en place dans le cadre du programme de sauvegarde de la brebis sicilosarde.

La recherche bibliographique a été accomplie en consultant les bases de données Scopus, Google Scholar et HAL. Les mots-clés suivants en français, et leur équivalent en anglais, ont été utilisés : élevage ovin tunisien, brebis laitière, race sicilo-sarde, zones d'élevage, performances zootechniques, qualité $\mathrm{du}$ lait, transformation fromagère, contraintes de développement, perspectives de relance.

\section{ORIGINE ET ZONES D'ÉLEVAGE}

La race sicilo-sarde tire son nom d'un croisement entre deux races provenant du Sud de l'Italie, considérées parmi les meilleures laitières ovines du bassin méditerranéen, à savoir: la comisana et la sarde, originaires respectivement de Sicile et de Sardaigne. Au début du $20^{\mathrm{e}}$ siècle, des fermiers siciliens arrivent en Tunisie avec des animaux de races comisana et sarde pour satisfaire leurs propres besoins en fromages. Ils s'installent sur les collines du Nord tunisien, autour du gouvernorat de Béja et de la délégation de Mateur relevant du gouvernorat de Bizerte (Figure 1). Cette population animale a permis de fixer la race sicilosarde (Aurejac et al., 2007) qui continue aujourd'hui à être rencontrée principalement au Nord du pays. Le climat subhumide caractéristique de ces régions est favorable à une production fourragère importante

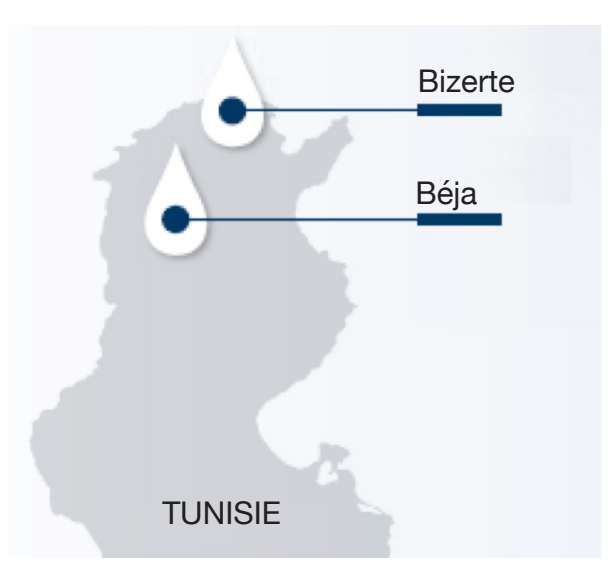

Figure 1. Zones d'élevage de la race sicilo-sarde en Tunisie - Areas for breeding of the Sicilo-Sarda breed in Tunisia. 
et un bon développement des prairies naturelles. Les informations relatives au schéma de ce croisement sont rares et difficiles à vérifier.

La production fromagère, exclusivement fermière au départ pour satisfaire les besoins en autoconsommation (Khaldi \& Farid, 1981), a évolué vers une transformation artisanale de plus grande ampleur, offrant des produits tels que les fromages sicilien et ricotta. Actuellement, le cheptel de la brebis sicilosarde est détenu aussi bien par des fermes du secteur organisé (fermes de l'État) que par des éleveurs privés (68\% ; Mohamed, 2008), essentiellement autour de Béja.

\section{3. ÉVOLUTION DES EFFECTIFS}

Jusqu'aux années 1990, la brebis sicilo-sarde n'était pas menacée. Depuis lors, les effectifs de cette race ont fortement chuté (Figure 2) en passant de 219000 à 37000,20000 et 8500 Unités Femelles, respectivement en 1995, 2000 et 2005 (Ouhichi, 2014). Les raisons de ce déclin rapide sont multiples : la privatisation des fermes de l'État converties en Sociétés de Mise en Valeur et de Développement Agricole (SMVDA), délaissant les productions les moins rentables; les encouragements de l'État favorisant essentiellement l'élevage bovin laitier ; la diminution des performances consécutive à des problèmes de suivi de la race (consanguinité élevée, pas de contrôle de la production ni des caractères phénotypiques d'intérêt, conduite non appropriée des troupeaux); la situation de monopole de l'industrie de transformation contribuant à la stagnation des prix et causant l'abandon fréquent de cette spéculation; l'adoption du croisement d'absorption de la race sicilo-

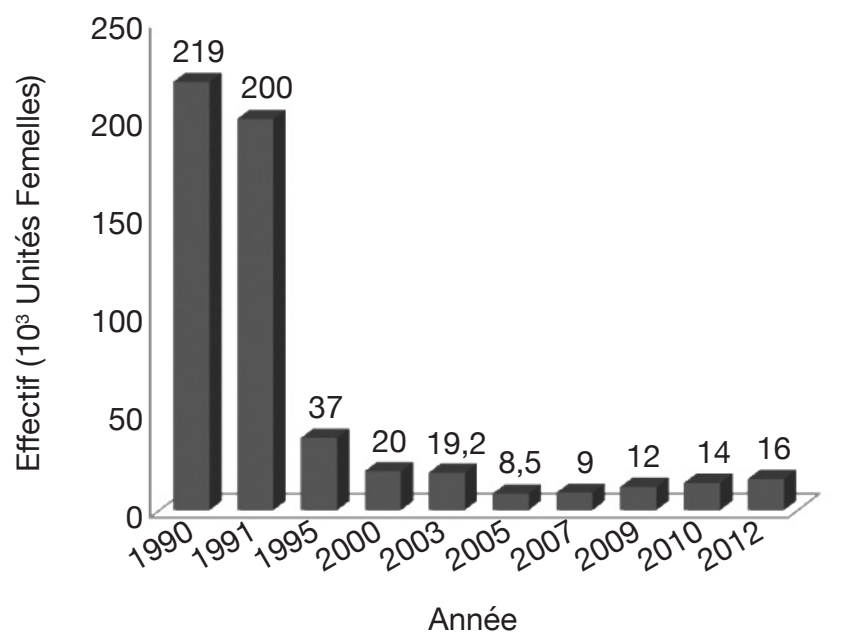

Figure 2. Évolution des effectifs de la race sicilo-sarde en Tunisie - Evolution of the numbers of the Sicilo-Sarda breed in Tunisia (Ouhichi, 2014). sarde par les races à viande et, enfin, la production de fromages non différenciés du lait de vache pour plus de quantité et de rentabilité immédiate aux dépens de produits de tradition et de plus forte typicité.

La privatisation a également conduit à la perte des meilleurs troupeaux détenus par les Unités Coopératives de Production Agricole (UCPA) et qui constituaient la base de sélection utilisée pour le programme d'amélioration génétique de cette race. Un effort considérable d'amélioration génétique, entrepris depuis les années 1970 par l'Office de l'Élevage et des Pâturages (OEP), a ainsi été anéanti.

À partir de 2005, la population semble amorcer une nouvelle augmentation régulière (Figure 2), à la suite de l'organisation de la profession (création des Groupements de Développement Agricole des éleveurs des brebis Sicilo-Sarde, GDA-SS). Ceci a notamment permis une meilleure organisation de l'opération de vente du lait, associée à une révision à la hausse du prix de vente et une intensification des programmes d'amélioration pour exploiter au mieux le potentiel de production des brebis.

\section{CARACTÉRISTIQUES D'ÉLEVAGE}

\subsection{Description morphologique}

Sur le plan de la configuration générale, la race sicilosarde est caractérisée par une taille moyenne au garrot variant entre 70 et $80 \mathrm{~cm}$ (Figure 3). Le poids moyen de l'adulte est de $45 \mathrm{~kg}$ chez la femelle et de 65 à $75 \mathrm{~kg}$ chez le mâle. Cette race a une couleur de robe très variable, pouvant être blanche, noire ou tachetée (Figure 4). La forme de la toison est hétérogène et jarreuse. La tête est légèrement allongée. Le profil est droit avec une légère courbure, la queue est fine et longue et les membres sont fins. Les cornes sont de petite taille et plus longues chez les mâles que chez

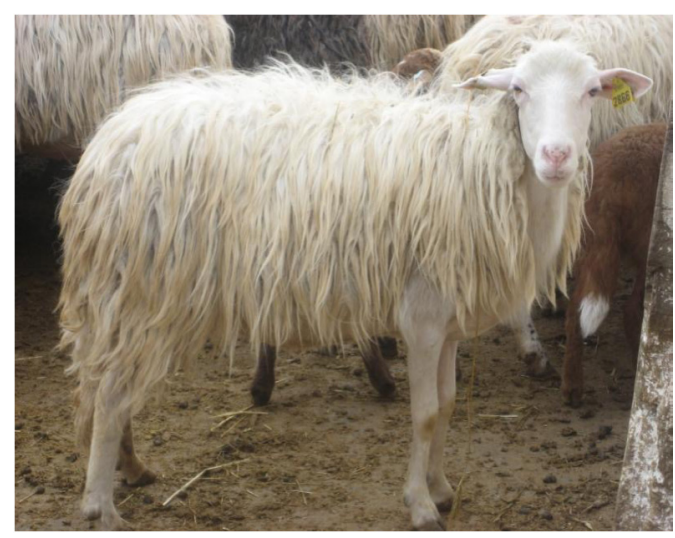

Figure 3. Brebis de race sicilo-sarde - Sicilo-Sarda dairy ewes (Rekik et al., 2005). 


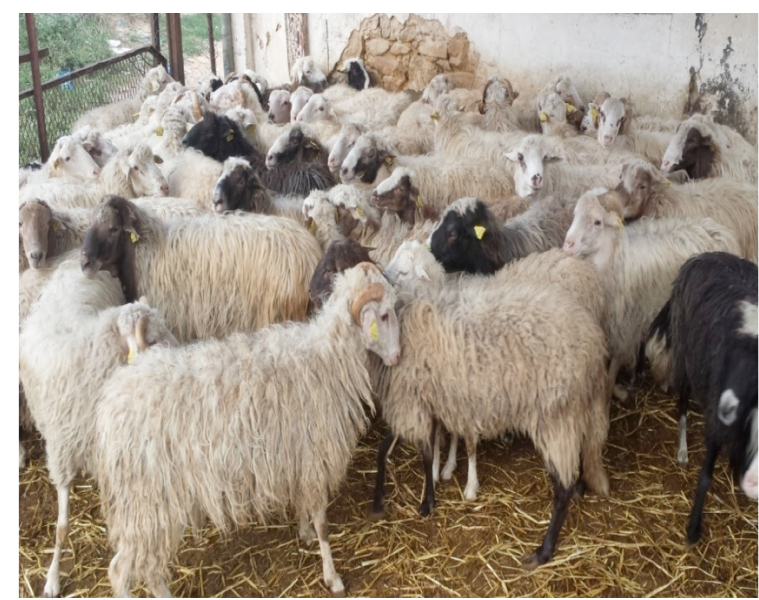

Figure 4. Jeunes béliers de race sicilo-sarde à l'UCPA Methline, Bizerte, Tunisie - Young rams of Sicilo-Sarda breed at UCPA Methline, Bizerte, Tunisia.

les femelles. Les oreilles sont petites et horizontales et le chanfrein est droit. L'encolure est plus ou moins longue. Le tronc est allongé et l'abdomen est ample (Rekik et al., 2005).

La glande mammaire est assez bien développée et dotée d'une forte attache avec des trayons droits (Khaldi \& Farid, 1981). Ses morphologies externe et interne n'ont été étudiées qu'une seule fois (Ayadi et al., 2011). Les principales caractéristiques morphologiques mammaires de cette race sont présentées dans le tableau 1. En raison du faible effectif des femelles choisies par ces auteurs au sein d'un seul élevage (relevant du secteur organisé) de la région de Mateur (Bizerte), ces résultats ne sont pas représentatifs de la population tunisienne et mériteraient qu'un programme plus large soit conduit pour la qualifier de façon plus exhaustive afin de gagner en représentativité.

Tableau 1. Caractères morphologiques mammaires de la brebis laitière sicilo-sarde à Bizerte, Tunisie - Mammary morphological traits of the Sicilo-Sarda dairy ewes in Bizerte, Tunisia (Ayadi et al., 2011).

\begin{tabular}{ll}
\hline Caractère mesuré & $\begin{array}{l}\text { Moyenne } \\
\text { (écart-type) }\end{array}$ \\
\hline $\begin{array}{l}\text { Angle moyen d'insertion du trayon } \\
\text { (degré) }\end{array}$ & $45,20( \pm 10,00)$ \\
\hline Longueur moyenne du trayon $(\mathrm{mm})$ & $18,50( \pm 4,90)$ \\
\hline Diamètre moyen du trayon $(\mathrm{mm})$ & $10,00( \pm 2,10)$ \\
\hline Distance entre trayons $(\mathrm{cm})$ & $7,50( \pm 1,10)$ \\
\hline Profondeur de la mamelle $(\mathrm{cm})$ & $5,04( \pm 0,14)$ \\
\hline Circonférence de la mamelle $(\mathrm{cm})$ & $38,20( \pm 3,40)$ \\
\hline Surface de la citerne $\left(\mathrm{cm}^{2}\right)$ & $11,60( \pm 4,50)$ \\
\hline
\end{tabular}

\subsection{Conduites d'élevage}

Les conduites d'élevage de la race sicilo-sarde sont plutôt traditionnelles avec une exploitation de type mixte (lait et viande). Les animaux sont généralement conduits dans deux types de système de production contrastés : le semi-intensif et l'extensif. Le premier est pratiqué pour les grands troupeaux (200 à 300 brebis), c'est le cas des exploitations étatiques et des grandes fermes privées. Dans ce système, l'alimentation est basée sur le pâturage (chaumes, orge, parcours), avec un recours fréquent aux foins, aux pailles et aux concentrés pendant les périodes de soudure et pendant la période de lactation (Rouissi et al., 2008b). Le second système se limite aux troupeaux de petite taille comptant 10 à 20 têtes. Il est caractérisé surtout par un faible niveau d'alimentation, basé essentiellement sur les ressources pastorales naturelles avec une complémentation très limitée en concentrés fermiers (Rekik et al., 2005).

Les saillies sont naturelles et libres sur chaleurs induites à contre-saison au printemps par moyens zootechniques (effet bélier, flushing et tonte), elles durent entre deux et trois mois et demi avec, par conséquent, des agnelages d'automne qui s'étalent du mois d'aout au mois de novembre avec un pic en septembre-octobre (Djemali et al., 1995; Rouissi et al., 2001). Ce schéma est celui classiquement adopté pour les races à viande en Tunisie, dont l'objectif est la finition des agneaux au printemps (Atti, 1998). Selon cet auteur, cette conduite est associée à une lactation qui se prolonge de septembre à juin, divisée en une première période d'allaitement, de 60-75 à plus de 100 jours (sevrage tardif) et une période de traite exclusive qui débute souvent au mois de décembre et peut se prolonger jusqu' au mois de juin, correspondant ainsi avec la saison verte (Djemali et al., 1995). Ce mode de conduite n'est pas favorable à une production laitière importante. En effet, avec une lactation très longue pour une brebis, associée à un chevauchement avec un début de gestation à partir du printemps, une chute sensible de la production laitière est observée, avec un risque élevé d'amaigrissement des brebis risquant de compromettre la lactation suivante. De plus, la phase d'allaitement exclusif peut freiner le potentiel de la mamelle et expliquer, en partie, le faible effet de la conduite alimentaire sur la production laitière chez cette race (Mohamed et al., 2008). La mise en place d'autres rythmes et saisonnalités de reproduction plus adaptés à la finalité laitière de cette race mériteraient donc d'être testés.

La transition vers la période de traite exclusive peut être brutale comme progressive (conduite mixte allaitement/traite pendant une courte période). La traite se fait ensuite deux fois par jour à un intervalle de $12 \mathrm{~h}$. Le recours à la monotraite est appliqué en fin de lactation lorsque le niveau de production laitière 
baisse. La traite est majoritairement manuelle, à l'exception de quelques éleveurs ayant des troupeaux de grande taille qui ont opté pour la traite mécanique, notamment en pot. Le manque de référentiel sur les différents systèmes de traite mécanisés pénalise la qualité et la quantité de lait collecté, alors qu'il s'agit d'un levier essentiel de la maitrise du travail de l'éleveur.

Un exemple d'organisation annuelle de conduite de la race sicilo-sarde en système semi-intensif est représenté au tableau 2.

\subsection{Performances de reproduction}

Peu d'études se sont intéressées aux caractéristiques de reproduction de la race sicilo-sarde en Tunisie. Lassoued et al. (2014) ont avancé qu'à l'instar des autres races ovines locales moyennement désaisonnées, la race sicilo-sarde exprime un maximum d'activité sexuelle en automne et en hiver, alors qu'elle diminue au printemps.

Concernant les performances reproductives, il est généralement admis que la race sicilo-sarde est fertile, mais moyennement prolifique. Les taux moyens de fertilité, de prolificité et de fécondité sont respectivement de 90,144 et $130 \%$. Ces performances sont relativement satisfaisantes, en comparaison avec les races ovines tunisiennes à viande. Une grande variabilité intra-ferme, essentiellement d'origine environnementale, a été mise en évidence (Selmi et al., 2009). Par ailleurs, une caractérisation préliminaire de la relation entre la production laitière et la reproduction des brebis sicilo-sardes (Aloulou et al., 2011), confortée par les travaux de Meraï et al. (2014), a permis de confirmer l'antagonisme de ces deux caractères chez les ruminants laitiers (Bodin et al., 1999). Il faudra donc être attentif à ne pas détériorer les performances de reproduction lorsqu'on cherchera à améliorer la productivité laitière.

\subsection{Performances laitières}

Considérations générales. Il est important de signaler que la Tunisie a longtemps été déficitaire en lait. Une autosuffisance fragile a été réalisée en 1999, mais n'a pas résisté à une succession d'années difficiles à partir de 2010. En 2017, l'OEP a annoncé une production annuelle de 1408 millions de litres en 2016 dont 145 millions de litres ont été transformés en fromage, ce qui représente environ $15 \%$ du lait industrialisé et près de $10 \%$ de la production laitière totale (Hasni, 2016). Une grande partie de cette production provient d'élevages bovins hors sol, ce qui questionne la durabilité de tels systèmes. Il en va autrement pour la production de lait de brebis qui reste traditionnelle et attachée aux ressources pastorales (Rastoin et al., 2016). Les circuits de distribution et de commercialisation artisanaux du lait des petits ruminants sont mal connus et peu identifiés. La production annuelle de fromage de brebis est estimée à 250 t (Ouhichi, 2014), ce qui représente une infime partie de la production et de la consommation des fromages en Tunisie qui s'élève à environ 3,7 kg par habitant par an (APII, 2016), largement derrière les champions européens en la matière, en particulier les Français $(25,9$ kg ; CNIEL, 2017). Une grande marge de progrès existe ainsi pour couvrir une demande sans cesse croissante de la population tunisienne.

Estimation quantitative du lait produit durant la période d'allaitement. Certaines études relatives à l'estimation de la production laitière lors de l'allaitement ont été effectuées sur la race sicilo-sarde en Tunisie. Khaldi (1987) a montré que cette production est significativement influencée par le nombre d'agneaux élevés et l'âge au sevrage. Elle est estimée pour un sevrage à 42 et à 90 jours, respectivement à environ 50 et $102 \mathrm{~kg}$ chez les femelles allaitant un seul agneau, contre près de 66 et $134 \mathrm{~kg}$ pour les mères des

Tableau 2. Conduites d'élevage annuelles de la brebis sicilo-sarde en Tunisie - Annual management of the Sicilo-Sarda dairy ewes in Tunisia (Rekik et al., 2005).

\begin{tabular}{|l|l|l|l|l|l|l|l|l|l|l|l|l|}
\hline & Sept. & Oct. & Nov. & Déc. & Janvier & Février & Mars & Avril & Mai & Juin & Juillet & Aout \\
\hline Lutte & & & & & & & & & & & & \\
\hline Agnelage & & & & & & & & & & & & \\
\hline Allaitement & & & & & & & & & & & & \\
\hline Sevrage & & & & & & & & & & & \\
\hline Production laitière & & & & & & & & & & & & \\
\hline Pâturage & & & & & & & & & & & & \\
\hline Alimentation, chaumes & & & & & & & & & & & & \\
\hline Aliments concentrés & & & & & & & & & & & & \\
\hline Alimentation, foins/pailles & & & & & & & & & & & & \\
\hline
\end{tabular}


jumeaux. Plus récemment, Mohamed et al. (2008) ont confirmé l'importance de l'âge au sevrage, mais pas du nombre d'agneaux allaités. À partir de trois âges au sevrage distincts (précoce à 45 jours, progressif entre 45 et 90 jours et tardif à 90 jours), ils ont estimé cette production respectivement à 64,$6 ; 81,1$ et $109,2 \mathrm{~kg}$ pour les mères de simples et à 59,$7 ; 77,2$ et $111,9 \mathrm{~kg}$ pour les mères de doubles. Un travail d'optimisation de la durée de la phase d'allaitement, en vue d'une meilleure production de lait sans pénaliser la croissance future des jeunes, sera donc à mener.

Estimation quantitative du lait produit durant la période de traite exclusive. La production laitière moyenne de la race sicilo-sarde lors de la traite exclusive s'est toujours située entre 50 et 1001 par brebis, avec plus de 801 dans $90 \%$ des cas, mais pour des durées de traite très différentes (Mohamed, 2008). Cette quantité peut atteindre 1171 par brebis par lactation lorsque les agneaux sont sevrés précocement à l'âge de 42 jours (Khaldi, 1987).

La valeur moyenne de la production laitière dans les troupeaux inscrits au contrôle des performances varie d'un auteur à l'autre (Tableau 3). Cette variabilité a de nombreuses origines. L'étude des principaux facteurs du milieu affectant la production laitière chez la race sicilo-sarde montre des effets de l'âge de la brebis, du numéro de lactation, du stade de lactation, de la prolificité, des durées d'allaitement, de la traite exclusive et du facteur combiné troupeau, année et mois d'agnelage (Djemali et al., 1995 ; Moujahed et al., 2009 ; Atti et al., 2011).

Cette production situe donc la brebis sicilo-sarde au niveau de la classe des brebis les moins productives de la région méditerranéenne (Carta et al., 2009). On constate, en effet, que sa production journalière est limitée $\left(0,41\right.$ à $0,801 \cdot \mathrm{j}^{-1}$; Tableau 3), par rapport aux $1,761 \cdot \mathrm{j}^{-1}$ pour la Lacaune, $1,25 \mathrm{l} \cdot \mathrm{j}^{-1}$ pour la sarde et $1,0 \mathrm{l} \cdot \mathrm{j}^{-1}$ pour la comisana (Bougler, 1990). Ces deux dernières races, à l'origine de la race sicilo- sarde, montrent qu'il existe un potentiel et une marge importante de progrès pour la race locale tunisienne.

Ayadi et al. (2011) ont déterminé la répartition de la production laitière dans la mamelle après la traite (Tableau 4). La part du lait alvéolaire est approximativement la même que celle du lait citernal. Par ailleurs, la quantité moyenne du lait résiduel qui ne pourra être extraite que sous des conditions parfaites de traite aussi bien manuelle que mécanique (Marnet et al., 1998) est de 0,17 et $0,18 \mathrm{~kg} \cdot \mathrm{j}^{-1}$, soit $26 \%$ et $27 \%$ de la production laitière totale pendant la période de traite exclusive, respectivement pour le mode de sevrage précoce (45 jours) et tardif ( 90 jours) (Mohamed et al., 2008). Ce chiffre est considéré comme particulièrement élevé comparativement à ceux cités dans la littérature : 11 à $20 \%$ en moyenne pour toutes les races du pourtour méditerranéen, selon l'étude exhaustive du projet FAO M4, avant toute sélection sur la traite (Labussière, $1983)$; 13 à $24 \%$ chez les brebis à vocation viande des Préalpes du Sud qui sont non adaptées à la traite mécanique et dont une grande partie n'exprime pas de réflexe d'éjection du lait à la traite (Ricordeau et al., 1963 ; Labussière et al., 1969) ; $17 \%$ en race locale slovaque Tsigai et $9 \%$ en Lacaune (Mačuhová et al., 2012); $8 \%$ en race manchega (Peris et al., 1995). La conduite de la traite (pratiques d'éleveur, matériel et réglages) n'est donc pas optimale pour assurer une bonne stimulation des animaux en vue d'une éjection la plus complète possible du lait. Il reste là encore une

Tableau 4. Répartition du lait dans la mamelle chez la brebis sicilo-sarde - Milk distribution in udder of SiciloSarda dairy ewes (Ayadi et al., 2011).

\begin{tabular}{ll}
\hline Caractère mesuré & Moyenne ( \pm écart-type) \\
\hline Lait alvéolaire $(\mathrm{ml})$ & $102( \pm 12)$ \\
\hline Lait citernal $(\mathrm{ml})$ & $120( \pm 14)$ \\
Pourcentage lait citernal $(\%)$ & $54( \pm 3)$ \\
\hline
\end{tabular}

Tableau 3. Caractéristiques de la production laitière chez la brebis sicilo-sarde en Tunisie - Characteristics for milk production of Sicilo-Sarda dairy ewes in Tunisia.

\begin{tabular}{lllll}
\hline Référence & $\begin{array}{l}\text { Durée de } \\
\text { l'allaitement }(\mathrm{j})\end{array}$ & $\begin{array}{l}\text { Durée de la } \\
\text { lactation }(\mathrm{j})\end{array}$ & $\begin{array}{l}\text { Production laitière } \\
\text { (traite exclusive) }(\mathrm{l})\end{array}$ & $\begin{array}{l}\text { Production laitière } \\
\text { journalière }\left(1 \cdot \mathrm{j}^{-1}\right)\end{array}$ \\
\hline Ben Hamouda \& Zitoun, 1988 & 127,5 & 116 & 57 & 0,49 \\
\hline Djemali et al., 1995 & 114,0 & 124 & 72 & 0,58 \\
\hline Rouissi et al., 2001 & - & $120-150$ & 96 & $0,64-0,80$ \\
\hline Moujahed et al., 2008 & - & 160 & 85 & 0,53 \\
\hline Aloulou et al., 2011 & - & 153 & 63 & 0,41 \\
\hline Meraï et al., 2014 & 100,4 & 132,8 & 61 & - \\
\hline Mâatoug et al., 2015 & 70 & 142 & 92 & 0,65 \\
\hline
\end{tabular}


marge de progrès importante, tant sur le plan technique et matériel de traite, que sur le plan de l'adaptation des mamelles à la machine à traire, à travers une sélection génétique incluant aussi des critères de morphologie fonctionnelle, à l'image de ce qui s'est fait pour la race Lacaune en France (Marie-Etancelin et al., 2005).

Qualité du lait produit. La composition moyenne du lait de brebis sicilo-sarde pour des élevages inscrits au contrôle laitier officiel est indiquée dans le tableau 5. La teneur moyenne en matière grasse est de 6,85\% (Bousselmi \& Othmane, 2015) contre 5,88, 6,50, 6,51 et $7,49 \%$ respectivement pour les races latxa, sarde, Lacaune et Churra. La teneur en protéines est de $6 \%$ contre $4,78,5,15,5,76$ et $6,11 \%$ respectivement pour les races manech à tête rousse, Lacaune, sarde et Churra (Carta et al., 2009), avec aussi $18 \%$ d'extrait sec total et $3,80 \%$ de lactose (Bousselmi \& Othmane, 2015). Cette composition chimique moyenne du lait de la race sicilo-sarde est donc dans la gamme haute des races laitières méditerranéennes. Ceci s'explique, en grande partie, par le faible niveau de production de cette race et, éventuellement, par la nature des ressources alimentaires, notamment pastorales (Atti et al., 2006). La richesse du lait de la brebis sicilo-sarde le destine exclusivement à la transformation fromagère (Ouhichi, 2014). Le rendement fromager moyen pour un fromage blanc est de 33,87 $\pm 6,10 \mathrm{~kg} \cdot 100 \mathrm{l}^{-1}$ (Bousselmi \& Othmane, 2015).

La composition chimique du lait varie aussi selon plusieurs facteurs liés à l'animal et à son environnement. Toutefois, l'alimentation, à travers la nature et le niveau des apports, reste le principal facteur qui influence la qualité chimique du lait (Atti et al., 2006 ; Rouissi et al., 2008a ; Moujahed et al., 2009; Atti, 2011). Le programme d'amélioration de la productivité de cette race devra veiller à maintenir autant que possible la richesse du lait, dont dépendent la qualité organoleptique et le rendement fromager.

Performances de croissance des agneaux. Les performances de croissance des agneaux de race sicilosarde demeurent faibles, comparativement aux races ovines tunisiennes à vocation viande. Ben Hamouda (1988) a montré que le Gain Moyen Quotidien entre 10 et 30 jours d'âge $\left(G M Q_{10-30}\right)$ des agneaux sicilosardes est de l'ordre de $118 \mathrm{~g} \cdot \mathrm{j}^{-1}$. Les performances des mâles sont supérieures à celles des femelles avec une différence de 5 et $6 \%$, respectivement, pour les caractères Poids à 30 jours d'âge $\left(P_{30}\right)$ et $G M Q_{10-30}$. Les performances des nés simples sont 30 et $34 \%$ supérieures à celles des nés doubles, respectivement pour ces deux mêmes critères zootechniques. Khaldi (1987) a révélé que la vitesse de croissance des agneaux sicilo-sardes, entre la naissance et l'âge de 42 jours, est voisine de $200 \mathrm{~g} \cdot \mathrm{j}^{-1}$ chez les simples et de $130 \mathrm{~g} \cdot \mathrm{j}^{-1}$ chez les doubles. Il a également observé qu'un sevrage précoce diminue sensiblement le poids vif des agneaux simples à l'âge de 90 jours. Il reste donc à évaluer la conduite alimentaire de ces jeunes en post-sevrage afin de compenser ce retard, si une conduite en sevrage plus précoce devait être adoptée.

\subsection{Schéma de sélection}

La race ovine sicilo-sarde n'a jamais fait l'objet d'un véritable schéma de sélection en Tunisie (Othmane, 2011). L'organisation de la micro-filière ovine laitière est dès lors une étape préalable et indispensable. Les efforts d'amélioration génétique, dont le maitre d'œuvre est l'OEP, se sont limités à l'identification et au contrôle des performances de quelques troupeaux du secteur organisé avec un effectif de 1500 à 3000 têtes par an avant 2005 (Rekik et al., 2005) et un effectif beaucoup plus faible et variable au cours des dix dernières années. Il existe aujourd'hui une insuffisance des moyens institutionnels pour pouvoir élargir cette base de sélection. Cette dernière s'est donc effectuée principalement sur la voie femelle basée sur une combinaison de la production laitière de la mère et la croissance entre 10 et 30 jours d'âge de l'individu à sélectionner. La conformation générale, celle des mamelles (évaluation globale sans guide et grille de notation) et la couleur unie de la robe sont également prises en compte lors du choix des antenaises destinées au renouvellement du troupeau.

Tableau 5. Composition moyenne du lait chez la brebis sicilo-sarde - Average milk composition of Sicilo-Sarda dairy ewes.

\begin{tabular}{|c|c|c|c|c|c|c|c|}
\hline \multirow[t]{2}{*}{$\begin{array}{l}\text { Composition du lait } \\
\left(\mathrm{g} \cdot \mathrm{l}^{-1}\right)\end{array}$} & \multicolumn{3}{|c|}{ Rouissi et al., 2008a $(n=28)$} & \multirow{2}{*}{$\begin{array}{l}\text { Moujahed et al., } \\
2009(n=804) \\
\text { Moyenne }\end{array}$} & \multicolumn{3}{|c|}{$\begin{array}{l}\text { Bousselmi \& Othmane, } 2015 \\
(\mathrm{n}=3260)\end{array}$} \\
\hline & Moyenne & Minimum & Maximum & & Moyenne & Minimum & Maximum \\
\hline Extrait sec total & 189,80 & 174,00 & 208,00 & - & 180,70 & 114,60 & 271,30 \\
\hline Matière grasse & 74,90 & 62,00 & 90,00 & 75,00 & 68,50 & 20,40 & 145,70 \\
\hline Matières azotées totales & 65,50 & 60,79 & 74,25 & 63,50 & 59,30 & 30,40 & 93,00 \\
\hline Lactose & 38,90 & 32,10 & 46,30 & - & - & - & - \\
\hline Matière minérale & 10,40 & 8,80 & 13,50 & - & - & - & - \\
\hline
\end{tabular}


Du côté des mâles, les individus les plus prometteurs sont sélectionnés à travers un projet dit «élèvesbéliers » géré par l'OEP. À l'âge de six mois, les béliers sont élevés dans des centres d'hébergement et ensuite diffusés à l'âge de 18 mois auprès de la base de sélection, comme des autres élevages, tout en veillant à bien maitriser la consanguinité qui affecte cette population à petit effectif. Pour minimiser les effets de cette consanguinité, un croisement d'amélioration, dit de « retrempe », est réalisé en faisant appel périodiquement à des semences fraiches issues de béliers sardes importés d'Italie. Djemali et al. (2009) ont évoqué le cas particulier du recours à des inséminations intra-utérines sur la période 2005-2007 avec des semences congelées sardes importées d'Italie en vue de pallier la rareté des géniteurs. Une relance de la variabilité phénotypique a été constatée principalement au niveau des troupeaux qui ont le plus bénéficié de ce croisement. Ces animaux, de types génétiques différents, ont profité de la supériorité de la race sarde qui a amélioré plusieurs critères de production ou de conformation. Bedhiaf-Romdhani et al. (2013) ont évoqué l'idée d'appliquer un schéma de sélection des béliers candidats, basé sur leur génotypage, pour minimiser les effets de la consanguinité.

Par ailleurs, la durée de lactation a été standardisée en fonction des conditions réelles d'élevage de la race et conformément aux normes internationales en vigueur, de façon à permettre un choix plus fiable des reproducteurs améliorateurs sur une base de comparaison commune. La précision des opérations du contrôle laitier a été étudiée à travers la simulation et l'évaluation de plusieurs scénarios de contrôle laitier simplifié (Othmane et al., 2006 ; Othmane \& Trabelsi, 2007). Othmane (2004) a par ailleurs estimé des paramètres génétiques (héritabilité et répétabilité) dont leurs valeurs sont assez faibles $\left(\mathrm{h}^{2}=0,11 ; \mathrm{r}=0,31\right)$ pour la production laitière chez cette race.

Ces initiatives individuelles en faveur de la sélection de la race sicilo-sarde devraient être rassemblées dans un programme fédérateur et plus global en vue de soutenir le regain d'intérêt observé actuellement pour cette race.

\section{TRANSFORMATION ET VALORISATION DU LAIT}

En aval de la filière, l'activité de fabrication fromagère en lait de brebis existe en Tunisie depuis longtemps. La première installation date des années 1950. Il s'agit d'une laiterie privée, la Société Tunisienne Laitière et Fromagère (SOTULAIFROM) construite à Mateur (gouvernorat de Bizerte) par des industriels fromagers de roquefort. Elle a démarré avec un savoir-faire dans la production de bleus de brebis type «roquefort» (Aurejac et al., 2007).
L'analyse de l'aval de la filière, basée sur l'étude des circuits de transformation et de commercialisation du lait et de ses dérivés, montre que $56 \%$ des éleveurs de brebis sicilo-sarde intègrent une filière industrielle et $22 \%$ transforment artisanalement leur lait en fromage et en ricotta (Mohamed, 2008). $\mathrm{Au}$ cours de ces dernières années, de nouvelles fromageries artisanales de petite taille ont été créées à Béja (LACTIMED, 2013 ; Rastoin et al., 2016) pour approvisionner un marché local en forte demande. La proximité de grandes agglomérations appuie la logique d'une transformation sur le territoire comme facteurs d'identité des productions et de durabilité.

\section{CONTRAINTES ET PERSPECTIVES DE RE- DÉVELOPPEMENT DE LA SICILO-SARDE}

\subsection{Menaces}

Comme cela a déjà été mentionné, les effectifs de la race ont chuté pour diverses raisons (manque de soutien, fragilité de la filière, désintérêt de la profession, etc.). De plus, la fragilité des systèmes d'élevage privé, résultant d'un faible niveau de technicité, entraine des conduites inadaptées des troupeaux. Enfin, même au sein du secteur organisé, les performances restent tributaires de la disponibilité de la main-d'œuvre spécialisée et de l'adoption limitée de nouvelles techniques d'élevage plus efficientes.

\subsection{Actions engagées et perspectives de relance}

Depuis 2005, l'élevage de brebis laitières en Tunisie a connu un regain d'intérêt de la part des éleveurs comme des instances techniques et politiques. Un programme de sauvegarde a été mis en place, impliquant toute l'interprofession (éleveurs, recherche, développement). Ce programme s'est basé notamment sur l'organisation des producteurs par la création en 2003 des GDA-SS. En effet, cette micro-filière a été réfléchie comme un espace pour voir émerger une nouvelle organisation professionnelle des filières agricoles, ainsi que pour une réflexion sur une adaptation des aides publiques qui en découlent (Rastoin et al., 2016).

Des améliorations seraient possibles moyennant l'instauration de changements dans les pratiques: intensification de la production par la création de surfaces fourragères dédiées aux ovins, élaboration d'un schéma de sélection adapté, généralisation de la traite mécanique et installation de centres de collecte de lait pour faciliter la commercialisation et la transformation des produits.

Une meilleure maitrise de la technicité de la production ovine laitière est impérative. Celle-ci ne peut être atteinte que dans le cadre d'une approche 
systémique impliquant tous les maillons de la microfilière. A titre d'exemple, l'amélioration génétique pourrait se réaliser par un recours plus soutenu à l'insémination artificielle et l'importation de béliers sardes, ainsi que par une meilleure valorisation des données phénotypiques d'importance.

De même, la sauvegarde de la race sicilo-sarde requiert le soutien des GDA-SS. Cela peut se matérialiser au travers des subventions suffisantes et des facilités d'octroi du matériel aux centres de collecte et aux industries laitières. D'autres incitants seront nécessaires, comme des soutiens à la reconstitution optimale des troupeaux, à la création d'autres usines de transformation industrielle et à l'incitation à l'élevage via des dons sous forme de petits troupeaux. La vulgarisation, l'encadrement et l'assistance des éleveurs sont aussi indispensables et sollicitent l'investissement accru des structures de développement agricole comme l'OEP et le ciblage de l'aide de l'État (Mohamed, 2008). En outre, la spécialisation en produits de terroirs pourrait assurer la pérennité de cette activité et dynamiser l'ensemble de la micro-filière sur les territoires avec ses retombées en matière d'emplois. La protection de ces produits par le dépôt de labels ou d'appellations d'origine protégées $(\mathrm{AOP})$ serait également à envisager. Enfin, le développement de l'agrotourisme pourrait faire partie des conditions de maintien de cette micro-filière, en la faisant découvrir et apprécier des gens de la ville comme des touristes.

\section{CONCLUSIONS}

La brebis sicilo-sarde est la seule race ovine laitière en Tunisie. Son niveau de production est actuellement limité mais avec un potentiel sous-valorisé. En effet, l'existence d'une variabilité individuelle importante des performances témoigne d'une marge importante de progrès possible. Un déclin prononcé de cette race a été enregistré à la fin des années 1990, mettant en péril l'existence d'une micro-filière ancrée depuis près d'un siècle dans le Nord du pays. À partir de 2005, une réorganisation en profondeur de la profession a fait émerger des signes de relance de la filière avec un regain d'intérêt pour cette race.

Pour remédier à son déclin, cette micro-filière ovine laitière a mis en avant ses atouts en matière de développement durable qui reposent à la fois sur une race locale particulièrement bien adaptée à son environnement et sur un savoir-faire de fabrication artisanale de fromages assurant la typicité des produits.

En consolidant des activités anciennes (agriculture et artisanat) et en en créant de nouvelles (fromageries et agrotourisme), cette micro-filière contribue au développement rural. Sur le plan économique, l'éleva- ge de la brebis sicilo-sarde favorise le maintien et la création d'activités et donc de l'emploi en zone rurale. En définitive, la gouvernance de la micro-filière reste soutenue par des structures professionnelles représentées par les Groupements de Développement Agricole. Un certain nombre de travaux de recherchedéveloppement (reproduction, alimentation, génétique, etc.) ont déjà été réalisés. D'autres études restent à mener en les axant sur des finalités permettant d'assurer un développement solide et pérenne de la filière. L'exemple du développement de la traite mécanique via des caractérisations se rapportant à la morphophysiologie de la mamelle et des trayons associée à l'étude des réglages les plus appropriés permettant d'améliorer l'efficacité de la traite, reste un des axes privilégiés en ce moment pour la race sicilo-sarde. L'aspect moléculaire est également de plus en plus considéré (Ben Sassi-Zaidy et al., 2014; El Hentati et al., 2017). Les perspectives sont donc prometteuses pour sauvegarder ce patrimoine menacé que représente la brebis sicilo-sarde.

\section{Remerciements}

Les auteurs remercient vivement Mademoiselle Hania Hamdi, chercheur post-doctoral à l'Institut Supérieur Agronomique de Chott Mariem, Sousse, Tunisie, pour sa contribution précieuse dans la mise en forme de l'article.

\section{Bibliographie}

Aloulou R. et al., 2011. Caractérisation de la relation entre la production et la reproduction des brebis de race sicilo-sarde en Tunisie. In : Khlij E., Ben Hamouda M. \& Gabiña D., eds. Mutations des systèmes d'élevage des ovins et perspectives de leur durabilité. Zaragoza, Espagne: CIHEAM/IRESA/OEP, Options Méditerranéennes, Série A, 97, 155-156.

APII (Agence de Promotion de l'Industrie et de l'Innovation), 2016. Fromagerie, http://www.tunisieindustrie.nat.tn/fr/ download/fichesPro/IAA/12.pdf, (22/01/2018).

Atti N., 1998. Effet du mode de conduite et de l'âge au sevrage de l'agneau sur les performances de production de la race laitière sicilo-sarde. Ann. INRAT, 71, 237-249.

Atti N., 2011. Système optimum de conduite des ovins : cas des conditions alimentaires améliorées du sud de la Méditerranée. In: Khlij E., Ben Hamouda M. \& Gabiña D., eds. Mutations des systèmes d'élevage des ovins et perspectives de leur durabilité. Zaragoza, Espagne: CIHEAM/IRESA/OEP, Options Méditerranéennes, Série A, 97, 51-60.

Atti N.,Rouissi H. \& Othmane M.H.,2006. Milk production, milk fatty acid composition and conjugated linoleic acid (CLA) content in dairy ewes raised on feedlot or grazing pasture. Livest. Sci., 104, 121-127. 
Atti N., Othmane M.H., Haider M. \& Toukebri M., 2011. Effets des saisons de lutte et d'agnelage sur les performances laitières et de reproduction des brebis de race sicilo-sarde. Ann. INRAT, 84, 157-167.

Aurejac R., Mercier G. \& Parguel P., 2007. Étude de la filière de l'élevage ovin laitier en Tunisie. Rapport final. Paris : Bureau de Coopération Technique Internationale/ Institut de l'Élevage.

Ayadi M. et al., 2011. Relationship between mammary morphology traits and milk yield of Sicilo-Sarde dairy sheep in Tunisia. Small Ruminant Res., 96, 41-45.

Bedhiaf-Romdhani S., Kaddach I., Bedhiaf A. \& Djemali M., 2013. Use of microsatellite markers for parentage identification and inbreeding control in Sicilo-Sarde dairy sheep flocks. In: the $8^{\text {th }}$ International Seminar, Technology creation and transfer in small ruminants: roles of research, development services and farmer associations, 11-13 June 2013, Tangier, Marocco. Roma:FAO; Montpellier, France: CIHEAM.

Ben Hamouda M., 1988. Effet du milieu sur le poids à 30 jours et le $\mathrm{GMQ}_{10-30}$ des agneaux de la race sicilosarde. Échos Rech. Agric., 2(1).

Ben Hamouda M. \& Zitoun K., 1988. Effet du milieu sur la quantité moyenne de lait par jour de traite en race sicilosarde. Revue INAT, 3(1), 81-90.

Ben Sassi-Zaidy Y., Maretto F., Charfi-Cheikrouha F. \& Cassandro M., 2014. Genetic diversity, structure, and breed relationships in Tunisian sheep. Small Ruminant Res., 119, 52-56.

BNG (Banque Nationale de Gènes), 2018. Groupe ressources génétiques animales, http://www.bng.nat. tn/index.php/wf-menu-config/reseau-de-la-bng/grressources-genetiques-animales, (22/04/2018).

Bodin L. et al., 1999. Génétique de la reproduction chez les ruminants. INRA Prod. Anim., 12(2), 87-100.

Bougler J., 1990. Confrontation internationale de races de brebis laitières méditerranéennes. In : Bougler J. \& Tisserand J.-L., eds. Les petits ruminants et leurs productions laitières dans la région méditerranéenne. Montpellier, France : CIHEAM, Options Méditerranéennes, Série A, 12, 9-18.

Bousselmi H. \& Othmane M.H., 2015. Milk composition and cheese making ability in ewes: influence of major milk components and $\mathrm{pH}$ on individual cheese yield at a laboratory scale in ewes. Greener J. Biol. Sci., 5(1), 001-009.

Carta A., Casu S. \& Salaris S., 2009. Invited review: current state of genetic improvement in dairy sheep. J. Dairy Sci., 92, 5814-5833.

CNIEL, 2017. L'économie laitière en chiffre. Paris : Centre National Interprofessionnel de l'Économie Laitière (CNIEL).

Djemali M., Ben M'Sallem I. \& Boraoui R., 1995. Effet du mois, mode et âge d'agnelage sur la production laitière des brebis sicilo-sardes en Tunisie. In : Caja G., Djemali M., Gabiña D. \& Nefzaoui A., eds. L'élevage ovin en zones arides et semi-arides. Saragosse, Espagne : CIHEAM, Options Méditerranéennes, Série A, 6, 111-117.

Djemali M., Bedhiaf-Romdhani S., Iniguez L. \& Inounou I., 2009. Saving threatened native breeds by autonomous production, involvement of farmers' organization, research and policy makers: the case of the Sicilo-Sarde breed in Tunisia, North Africa. Livest. Sci., 120, 213-217.

El Hentati H. et al., 2017. Molecular polymorphism of the Sicilian-Sardinian dairy sheep in the Nagachia region in Tunisia. IJPAES, 7(2), 36-39.

FAO, 2007. Biodiversité et animaux d'élevage, https:// www.pyrenees-pireneus.com/Agriculture-Pyrenees/ Pastoralisme-Pyrenees/Biodiversite-Environnement/ Pastoralisme-BiodiversiteAnimale.htm, (25/10/2017).

Haenlein G.F.W., 2007. About the evolution of goat and sheep milk production. Small Ruminant Res., 68, 3-6.

Hasni M., 2016. La filière lait en Tunisie, http://avfa.agrinet. tn/upload/supports_ar/jammel1.pdf, (22/01/2018).

Khaldi G., 1987. Influence de l'âge au sevrage et du mode de naissance des agneaux sur la production laitière des brebis de race sicilo-sarde pendant les phases d'allaitement et de traite. Ann. INRAT, 60.

Khaldi G. \& Farid M., 1981. Encyclopédie des productions animales dans le monde arabe. Cas de la République tunisienne. Damas : Arab Center for the Studies of Arid zones and Dry lands (ACSAD).

Labussière J., 1983. Étude des aptitudes laitières et de la facilité de traite de quelques races de brebis du Bassin Méditerranéen. In: $3^{e}$ Symposium International de Traite mécanique des petits ruminants. Valladolid, Espagne: Édition Sever Cuesta, 730-794.

Labussière J. et al., 1969. Importance, composition et signification des différentes fractions de lait obtenues successivement au cours de la traite mécanique des brebis. Ann. Zootech., 18(2), 185-196.

LACTIMED, 2013. Valorisation des produits laitiers typiques de Bizerte et Béja. Diagnostic et stratégie locale, http://www.animaweb.org/sites/default/files/lac_ rapportdiagnostic_tunisie_fr_final.pdf, (18/01/2018).

Lassoued N., Rekik M., Khenissi S. \& Merai A., 2014. Seasonality of oestrus, ovulation and ovulation rate of Sicilo-Sarde sheep. J. Anim. Physiol. Anim. Nutr., 98(4), 686-692.

Mâatoug S., Aouechri A., Abidi M. \& Marzouki M.L., 2015. Conduite de l'élevage ovin laitier en Tunisie: contraintes et possibilités d'amélioration. Nat. Technol. Ser. $B, \mathbf{1 2}, 11-15$.

Mačuhová L., Tančin V., Uhrinča M. \& Mačuhová J., 2012. The level of udder emptying and milk flow stability in Tsigai, improved Valachian, and Lacaune ewes during machine milking. Czech J. Anim. Sci., 57(5), 240-247.

Marie-Etancelin C. et al., 2005. Première évaluation génétique de la morphologie mammaire des brebis laitières Lacaune. In: Actes des $12^{e}$ Rencontres Recherches Ruminants, Paris, 18 décembre 2005, 289292. 
Marnet P.G., Negrao J.A. \& Labussière J., 1998. Oxytocin release and milk ejection parameters during milking of dairy ewes in and out of natural season of lactation. Small Ruminant Res., 28, 183-191.

Meraï A. et al., 2014. Non-genetic sources of variation of milk production and reproduction and interactions between both classes of traits in Sicilo-Sarde dairy sheep. Animal, 8(9), 1534-1539.

Mohamed A., 2008. L'élevage ovin laitier en Tunisie: analyse de la situation actuelle, contraintes, moyens et perspectives de développement. Thèse de doctorat: Institut National Agronomique de Tunisie, Tunis (Tunisie).

Mohamed A., Khaldi S., Rekik B. \& Khaldi G., 2008. Normal and residual milk yields in Sicilo-Sarde ewes: effects of litter size and the weaning age of lambs. Res. J. Anim. Sci., 2(5), 144-148.

Moujahed N., Jounaidi A., Kayouli C. \& Damergi C., 2008. Effects of management system on performances of the Sicilo-Sarde ewes farmed in Northern Tunisia. In : Papachristou T.G., Parissi Z.M., Ben Salem H. \& Morand-Fehr P., eds. Nutritional and foraging ecology of sheep and goats. Zaragoza, Spain: CIHEAM/FAO/ NAGREF, Options Méditerranéennes, Série A, 85, 393 397.

Moujahed N. et al., 2009. Analyse des principaux facteurs de variation de la production laitière et de la composition du lait chez la brebis sicilo-sarde dans la région de Béja (Tunisie). Livest. Res. Rural Dev., 21(4).

OEP(Office de l'Élevage et des Pâturages), 2016.Indicateurs du secteur de l'élevage en Tunisie 2015. Rapport annuel. Tunis : OEP.

OEP (Office de l'Élevage et des Pâturages), 2017. Données sectorielles 2016, productions, http://www.oep.nat. tn/index.php/fr/donnees-sectorielles/41-productions, $(01 / 11 / 2017)$.

Othmane M.H., 2004. Estimation des paramètres génétiques de la production laitière avec le modèle animal du jour de contrôle "test-day model" chez la brebis sicilo-sarde. Ann. INRAT, 77, 171-190.

Othmane M.H., 2011. Amélioration génétique de la race ovine sicilo-sarde : aspects organisationnels et acquis de recherches des cinq dernières années sur le troupeau de l'INRAT. In : Khlij E., Ben Hamouda M. \& Gabiña D., eds. Mutations des systèmes d'élevage des ovins et perspectives de leur durabilité. Saragosse, Espagne: CIHEAM/IRESA/OEP, Options Méditerranéennes, Série A, 97, 157-160.

Othmane M.H., Trabelsi L. \& Aloulou R., 2006. Standardisation de la production du lait des brebis SiciloSarde en tenant compte des conditions de production et des normes en vigueur. Ann. INRAT, 79, 165-178.
Othmane M.H. \& Trabelsi L., 2007. Suitability of simplified methods for monthly and twice monthly milk recording, and new predicting factors for the alternate a.m./p.m. method in dairy ewes under low input production system. J. Dairy Res., 74, 406-411.

Ouhichi R., 2014. Dispositifs d'appui à l'innovation et au développement territorial en Tunisie : cas de la brebis Sicilo-Sarde - Tunisie. In : $2^{e}$ Séminaire Méditerranéen LACTIMED, 5 mai 2014, Zahlé, Liban.

Peris C. et al., 1995. Examination of systems that exert traction on the teatcup and reduce teat bending in machine milking of ewes. Ann. Zootech., 44, 49-58.

Piras M., Ligios S., Sitzia M. \& Fois N., 2007. Out of season sheep milk production in Sardinia. Ital. J. Anim. Sci., 6(1), 588-590.

Rastoin J.L., Zaite M. \& Ben Youssef Z., 2016. Contribution des systèmes alimentaires territorialisés à la résilience au changement climatique en région méditerranéenne. Application à un projet de cluster agroécologique dans la vallée de la Medjerda en Tunisie. CIHEAM Watch Lett., 36.

Rekik M., Aloulou R. \& Ben Hammouda M., 2005. Small ruminant breeds of Tunisia. In: Iniguez L., ed. Characterization of small ruminant breeds in West Asia and North Africa. Vol. 2: North Africa. ed. International Center for Agricultural Research in the Dry Areas, 91140 .

Ricordeau G. et al., 1963. Traite à la machine des brebis Préalpes du sud. Importance des différentes opérations de la traite. Ann. Zootech., 12(3), 203-223.

Rouissi H. et al., 2001. Performances zootechniques de la race ovine Sicilo-Sarde en Tunisie. In : Rubino R. \& Morand-Fehr P., eds. Production systems and product quality in sheep and goats. Zaragoza, Spain : CIHEAM, Options Méditerranéennes, Série A, 46, 231-236.

Rouissi H. et al., 2008a. Étude de la qualité du lait des ovins laitiers en Tunisie. In : Olaizola A., Boutonnet J.P. \& Bernués A., eds. Mediterranean livestock production: uncertainties and opportunities. Zaragoza, Spain : CIHEAM/CITA/CITA, Options Méditerranéennes, Série A, 78, 307-311.

Rouissi H. et al., 2008b. Performances laitières de la brebis Sicilo-Sarde tunisienne complémentée par un concentré local. Livest. Res. Rural Dev., 20(7), 102-108.

Selmi H. et al., 2009. Performances de reproduction et de production en relation avec l'état sanitaire des brebis laitières Sicilo-Sardes. Livest. Res. Rural Dev., 21(8). 